\title{
RECICLAGEM E INCORPORAÇÃO DE NUTRIENTES AO SOLO PELO CULTIVO INTERCALAR DE ADUBOS VERDES EM POMAR DE LARANJEIRA-'PÊRA'1
}

\author{
JOSÉ ANTONIO ALBERTO DA SILVA², GODOFREDO CESAR VITTI ${ }^{3}$, \\ EDUARDO SANCHES STUCHI ${ }^{2}$, OTÁVIO RICARDO SEMPIONATO²
}

\begin{abstract}
RESUMO - O experimento de campo foi conduzido na Estação Experimental de Citricultura de Bebedouro-SP (EECB), visando a avaliar a produção de matérias verde e seca e quantidade de nutrientes incorporados ao solo pelo cultivo intercalar de Crotalaria juncea, Crotalaria spectabilis, Cajanus cajan, Mucuna aterrima, Mucuna deeringiana, Dolichos labe-labe e Canavalia ensiformis, nos anos agrícolas de 1989/90, 90/91, 91/92 e 92/93, em pomar de laranjeira-'Pêra' (Citrus sinensis L. Osbeck), enxertada sobre tangerineira'Cleópatra' (Citrus reshi Hort.), plantada em maio de 1987 e espaçadas de 7x5 m, num Latossolo Vermelho-Escuro. A produtividade média das espécies plantadas sem adubações e cultivos pós-semeadura, nos quatro anos de estudo, foram: 6,$55 ; 1,23 ; 3,42 ; 1,78 ; 1,75$; 1,61 e 3,03 t/ha de matéria seca, respectivamente, considerando plantio apenas na área intercalar de citros ( $50 \%$ da área total). A análise química do material seco revelou a incorporação de volume considerável de $\mathrm{N}, \mathrm{P}_{2} \mathrm{O}_{5}, \mathrm{~K} 2 \mathrm{O}, \mathrm{Ca}, \mathrm{Mg}, \mathrm{S}, \mathrm{B}, \mathrm{Cu}, \mathrm{Fe}, \mathrm{Mn}$ e $\mathrm{Zn}$ pelas leguminosas, onde podemos considerar que parte do $\mathrm{N}$ é proveniente da fixação simbiótica do $\mathrm{N}_{2}$ atmosférico pelas leguminosas e $\mathrm{o}$ restante e demais nutrientes provenientes do importante processo de reciclagem de nutrientes do solo, que são absorvidos pelas leguminosas das camadas subsuperficiais e incorporados na superfície do solo, onde estarão novamente disponíveis às plantas cítricas. A $C$. juncea foi a espécie que se destacou como maior produtora de biomassa e incorporadora de nutrientes, seguida pelo $C$. cajan e C. ensiformis.
\end{abstract}

Termos para indexação: Citrus sinensis, leguminosas, Crotalaria juncea, Crotalaria spectabilis, Cajanus cajan, Mucuna aterrima, Mucuna deeringiana, Dolichos labe-labe, Canavalia ensiformis

\section{RECICLYING AND INCORPORATION OF NUTRIENTS TO THE SOIL IN ORCHARD OF 'PERA' ORANGE BY CULTIVATION WITH COVER CROPS}

\begin{abstract}
The experiment was carried out at the Experimental Station of Bebedouro-SP (EECB), to evaluate the production of green manure and incorporated nutrients to soil by inter-cropping Crotalaria juncea, Crotalaria spectabilis, Cajanus cajan, Mucuna aterrima, Mucuna deeringiana, Dolichos labe-labe and Canavalia ensiformis, in the agricultural years of 89/90, 90/91, 91/92 and 92/ 93, in the orchard of 'Pera' (Citrus sinensis L. Osbeck), grafted on Cleópatra mandarin (Citrus reshi Hort.), planted in May of 1987 and spaced at $7 \times 5 \mathrm{~m}$. The average crop of cultivated species in the four years of study was: 6,$55 ; 1,23 ; 3,42 ; 1,78 ; 1,75 ; 1,61$ and $3,03 \mathrm{t} / \mathrm{ha}$ of dry matter, respectively, considering the cultivation only at the middle of the citros orchard (50\% of the total area). The chemical analysis of the material, revealed a considerable incorporation of $\mathrm{N}, \mathrm{P}_{2} \mathrm{O}_{5}, \mathrm{~K} 2 \mathrm{O}, \mathrm{Ca}, \mathrm{Mg}, \mathrm{S}, \mathrm{B}, \mathrm{Fe}, \mathrm{Mn}$ and $\mathrm{Zn}$ by leguminous plants, where we consider a parcel of the $\mathrm{N}$ comes from the atmospheric symbiotic fixation in the leguminous plants and the remaining and other nutrients comes from the recycling process of nutrients of soil, that are absorbed by the leguminous plants of the sub-superficial and incorporated in surface of the soil, where they will be again available to citrus plants. $C$. juncea produced the highest amount of biomass and incorporation of nutrients, followed by the C. cajan and C. ensiformis.
\end{abstract}

Index term: Citrus sinensis, leguminous plant, Crotalaria juncea, Crotalaria spectabilis, Cajanus cajan, Mucuna aterrima, Mucuna deeringiana, Dolichos labe-labe, Canavalia ensiformis

\section{INTRODUÇÃO}

Adubação verde é a prática de cultivo e incorporação de plantas, produzidas no local ou adicionadas, com a finalidade de preservar e/ou restaurar os teores de matéria orgânica e nutrientes dos solos, indo ao encontro da tendência mundial da busca de alimentos mais saudáveis, provenientes da agricultura orgânica ou produzidos com a mínima utilização de insumos químicos e degradação do meio ambiente (Silva et al., 1999).
A principal vantagem do emprego de espécies leguminosas na adubação verde é reduzir a aplicação de nitrogênio via adubo químico, pois essas plantas fixam nitrogênio do ar, através de simbiose com bactérias do gênero Rhizobium, enriquecendo o solo com esse macronutriente.

O sistema radicular ramificado e profundo das leguminosas proporciona aumento na eficiência de utilização dos adubos, uma vez que trazem às camadas superficiais do solo nutrientes perdidos por lixiviação, principalmente potássio (K), 
cálcio $(\mathrm{Ca})$, magnésio $(\mathrm{Mg})$ e nitrato $\left(\mathrm{NO}_{3}\right)$, funcionando também como "agente minerador" dos nutrientes de pouca disponibilidade como o fósforo (P) e o molibdênio (Mo), tornando-os mais disponíveis às culturas subseqüentes. Além disso, as raízes de adubo verde e do mato fazem, por assim dizer, uma subsolagem biológica, criando pequenos canais no solo por onde circulam a água e o ar (Russell et al., 1981).

O material orgânico produzido, geralmente rico em macro e micronutrientes, aumenta a capacidade de troca catiônica do solo, a infiltração e a retenção de água no solo, tornando mais favoráveis as condições para o desenvolvimento microbiano no solo, tendo ainda algumas espécies efeitos alelopáticos sobre nematóides e plantas invasoras (Miyasaka et al., 1984).

Segundo Silva et al. (1999), os adubos verdes comportamse como uma planta daninha no pomar cítrico, pois podem competir por água, nutrientes, sol e pelo espaço aéreo e do solo. Quando bem usados, entretanto, esses inconvenientes pesam relativamente pouco, sendo compensados pelas vantagens que seu cultivo apresenta. Para os citros, devem ser escolhidas espécies que possuam sementes uniformes e de bom poder germinativo, com exigência relativamente baixa quanto ao preparo e fertilidade do solo, de rápido crescimento, precoce, fácil manejo, sistema radicular profundo, que dispensem tratos culturais, apresentem resistência a pragas e doenças e produzam grande quantidade de matéria seca.

As espécies utilizadas como adubo verde para citros dividem-se em plantas de verão, normalmente leguminosas plantadas no início das chuvas e manejadas até o final destas, e as de inverno (leguminosas e gramíneas), plantadas no final das chuvas e manejadas quando em pleno florescimento (Silva et al., 1999).

Tendo em vista o exposto, o presente experimento objetivou avaliar a produção de material vegetal, incorporação de nutrientes ao solo pela fixação de $\mathrm{N}_{2}$ atmosférico e reciclagem de N, P, K, Ca, Mg, S, B, Cu, Fe, Mn e Zn do solo, pelo cultivo intercalar de adubos verdes (leguminosas) em pomar de laranjeira'Pêra'.

\section{MATERIAL E MÉTODOS}

O experimento foi conduzido em condições de campo na Estação Experimental de Citricultura de Bebedouro - SP (EECB), situada na latitude $20^{\circ} 53^{\prime} 16^{\prime \prime} \mathrm{S}$, longitude $48^{\circ} 28^{\prime} 11^{\prime \prime} \mathrm{W}$ e altitude de $601 \mathrm{~m}$, com clima do tipo Cwa e solo classificado como
Latossolo Vermelho-Escuro, epieutrófico, endoálico, A moderado, textura média (Haplustox). A análise química inicial do solo indicou os seguintes valores nas profundidades de 0-20 e 21-40 cm, respectivamente: $\mathrm{pH}\left(\mathrm{CaCl}_{2}\right)$ 4,6 e 4,0; matéria orgânica (M.O.) em g/ $\mathrm{dm}^{3}=30$ e 26; P resina em mg $/ \mathrm{dm}^{3}=5$ e $3 ; \mathrm{em} \mathrm{mmol}_{c}$ $\mathrm{dm}^{3}: \mathrm{K}=1,2$ e 0,$7 ; \mathrm{Ca}=10,8$ e 5,6; $\mathrm{Mg}=6,1$ e 3,1; $\mathrm{H}+\mathrm{Al}$ (hidrogênio + alumínio $=38$ e 47 ; SB (soma de bases) $=18,1$ e 9,4; CTC (capacidade de troca de cátions) $=56,1$ e 56,4 e V\% $=32$ e 17 . Utilizou-se um pomar de laranjeira-'Pêra' (Citrus sinensis L. Osbeck) enxertada sobre tangerineira- 'Cleópatra' (Citrus reshi Hort.), plantada em maio de 1987 e espaçadas de 7,0 x 5,0 m.

O primeiro plantio das leguminosas intercalar à laranjeiraPêra' deu-se em novembro de 1989, com replantio das espécies nos anos de 1990, 1991 e 1992, ou seja, durante quatro anos agrícolas.

O delineamento experimental utilizado foi em blocos casualizados, com sete tratamentos ou leguminosas (Tabela 1) e quatro repetições, totalizando 28 parcelas de 20 metros de comprimento cada, com sete linhas de leguminosas espaçadas de $0,5 \mathrm{~m}$ e plantadas dos dois lados (entrelinhas) da 'Pêra'; portanto, o plantio das leguminosas ocupou $50 \%$ da área implantada de 'Pêra'.

Não se utilizou adubação química para as leguminosas, nem mesmo cultivo pós-plantio, sendo as mesmas roçadas aos $113,148,107$ e 106 dias após o plantio, respectivamente de cada ano, ficando o material sobre a superfície do solo.

Antes do corte das leguminosas (roçagem), efetuaram-se amostragens de todo o material da parte aérea nas parcelas para determinação de peso verde e, posteriormente, peso seco. Estas amostras foram então analisadas quimicamente para a determinação dos teores de macro $(\mathrm{N}, \mathrm{P}, \mathrm{K}, \mathrm{Ca}, \mathrm{Mg}, \mathrm{S})$ e micronutrientes $(\mathrm{B}, \mathrm{Cu}, \mathrm{Fe}, \mathrm{Mn}$ e $\mathrm{Zn}$ ), segundo metodologia descrita por Malavolta et al. (1989).

Os resultados das análises químicas do solo em duas profundidades, nas linhas de leguminosas e nos citros, a incidência de plantas daninhas e cobertura do solo, bem como a produção dos citros podem ser encontrados em Silva (1995).

\section{RESULTADOS E DISCUSSÃO}

No primeiro plantio 1989/90, as espécies plantadas produziram após os 113 dias da semeadura, uma quantidade menor em peso de material verde e seco, quando se compara com os demais plantios (Tabela 2), sugerindo que o solo, neste ano, se

TABELA 1 - Espécies de leguminosas e densidade de sementes utilizadas na semeadura, EECB, Bebedouro-SP, 1989

\begin{tabular}{lccc}
\hline Espécies & Nome Comum & Sementes (kg/ha) & Densidade (sementes $/ \mathrm{ml}$ ) \\
\hline Crotalaria juncea L. & crotalária juncea & 30 & 25 \\
Crotalaria spectabilis Roth & crotalária spectabilis & 15 & 38 \\
Cajanus cajan (L.) Millsp & guandu & 50 & 17 \\
Mucuna aterrima & mucuna-preta & 85 & 5 \\
Mucuna deeringiana & mucuna-anã & 105 & 10 \\
Dolichos labe-labe L. & labe-labe & 60 & 13 \\
Canavalia ensiformis (L.) DC. & feijão-de-porco & 100 & 5 \\
\hline
\end{tabular}


TABELA 2 - Matéria verde e seca da parte aérea das leguminosas, anos agrícolas 1989/90 a 92/93, EECB, Bebedouro-SP

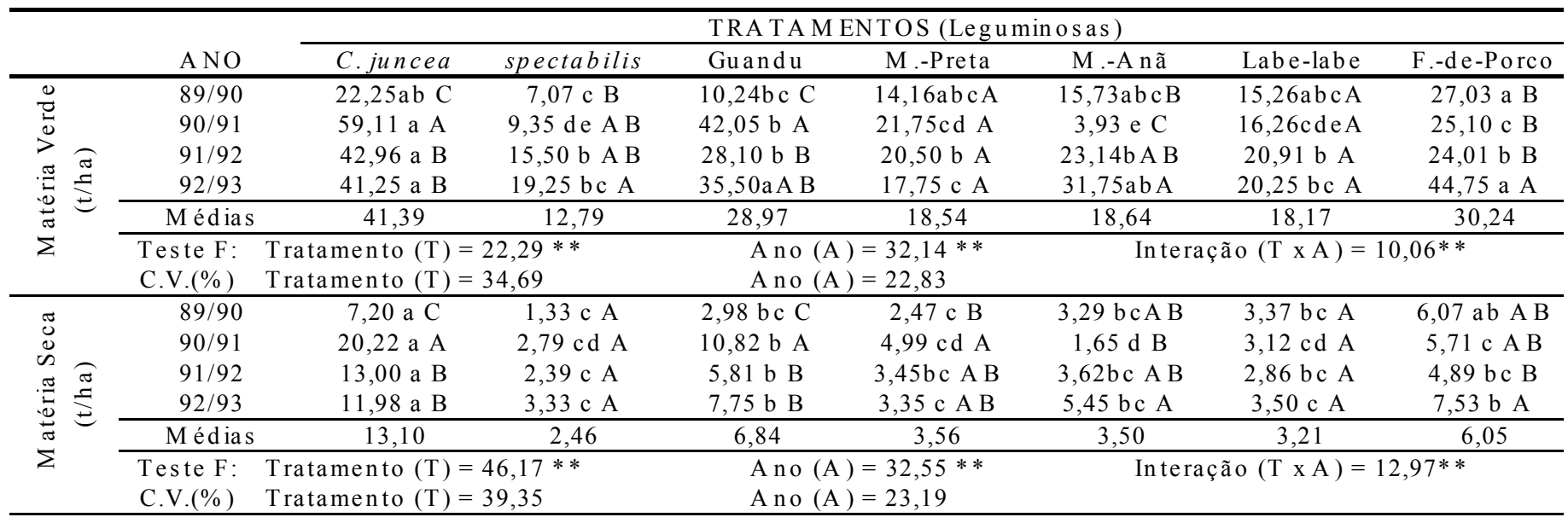

- Em cada ano (A), médias seguidas pela mesma letra maiúscula na vertical e em cada tratamento (T), médias seguidas pela mesma letra minúscula na horizontal, não diferem entre si, pelo teste de Tukey, a $5 \%$ de probabilidade $(\mathrm{P}>0,05)$

- Valores considerando plantio em $100 \%$ da área; para este caso de cultivo intercalar, considerar $50 \%$ dos valores acima.

TABELA 3 - Teores de macronutrientes da parte aérea das leguminosas (matéria seca), anos agrícolas 1989/90 a 92/93, EECB, Bebedouro-SP

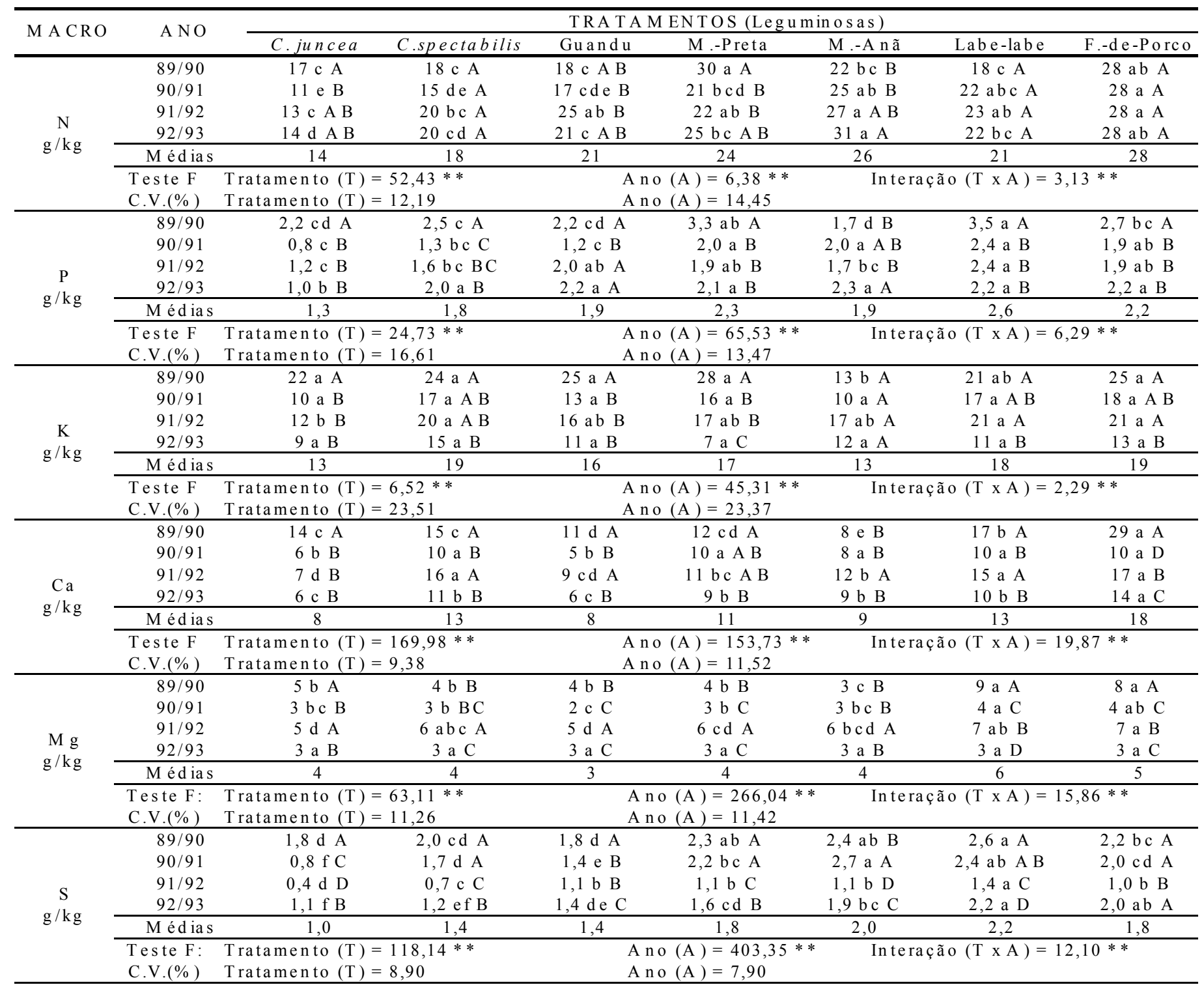

- Em cada ano (A), médias seguidas pela mesma letra maiúscula na vertical e em cada tratamento (T), médias seguidas de mesma letra minúscula na horizontal, não diferem entre si, pelo teste de Tukey, a $5 \%$ de probabilidade $(\mathrm{P}>0,05)$. 
TABELA 4 - Teores de micronutrientes da parte aérea das leguminosas (matéria seca), anos agrícolas 1989/90 a 92/93, EECB, BebedouroSP

\begin{tabular}{|c|c|c|c|c|c|c|c|c|}
\hline \multirow{2}{*}{ M ICRO } & \multirow{2}{*}{ A NO } & \multicolumn{7}{|c|}{ TRA TA M ENTOS (Leguminosas) } \\
\hline & & C. juncea & C.spectabilis & Guandu & M.-Preta & M.-A nã & Labe-labe & F.-de-Porco \\
\hline \multirow{6}{*}{$\begin{array}{c}\mathrm{B} \\
\mathrm{mg} / \mathrm{kg}\end{array}$} & $89 / 90$ & $17 \mathrm{~b} \mathrm{~B}$ & $22 \mathrm{ab} \mathrm{B}$ & $16 \mathrm{~b} \mathrm{~B}$ & $21 \mathrm{ab} B C$ & 26 a $\mathrm{B}$ & 26 a B & 26 a B \\
\hline & $90 / 91$ & $13 \mathrm{c} \mathrm{B}$ & 27 a $B$ & $16 \mathrm{bc} \mathrm{B}$ & 25 a B & $21 \mathrm{ab} B$ & 25 a B & 25 a B \\
\hline & $91 / 92$ & 19 b c A B & 35 a $\mathrm{A}$ & $19 \mathrm{bc} \mathrm{B}$ & $18 \mathrm{c} \mathrm{C}$ & $21 \mathrm{bc} \mathrm{B}$ & $26 \mathrm{~b} \mathrm{~B}$ & $22 \mathrm{bc} \mathrm{B}$ \\
\hline & $92 / 93$ & $25 \mathrm{~b} \mathrm{~A}$ & 36 a A & 42 a $\mathrm{A}$ & 41 a $\mathrm{A}$ & 35 a A & 38 a A & 42 a A \\
\hline & Médias & 18 & 30 & 23 & 26 & 26 & 29 & 28 \\
\hline & $\begin{array}{l}\text { Teste F: } \\
\text { C.V. }(\%)\end{array}$ & \multicolumn{2}{|c|}{$\begin{array}{l}\text { Tratamento }(\mathrm{T})=23,08 * * \\
\text { Tratamento }(\mathrm{T})=12,83\end{array}$} & \multicolumn{3}{|c|}{$\begin{array}{l}\text { A no }(\text { A })=104,69 * * \\
\text { Ano }(A)=15,03\end{array}$} & ão $(\mathrm{T} \times \mathrm{A})=$ & $4,93 * *$ \\
\hline \multirow{7}{*}{$\begin{array}{c}\mathrm{Cu} \\
\mathrm{mg} / \mathrm{kg}\end{array}$} & $89 / 90$ & $9 \mathrm{c} \mathrm{A}$ & $12 \mathrm{bc} \mathrm{A}$ & $11 \mathrm{c} \mathrm{A}$ & $17 \mathrm{ab} \mathrm{A}$ & 2 a B & $12 \mathrm{bc} \mathrm{A}$ & $8 \mathrm{c} \mathrm{A}$ \\
\hline & $90 / 91$ & $7 \mathrm{~d} \mathrm{~A}$ & $12 \mathrm{c} \mathrm{A}$ & $10 \mathrm{~cd} \mathrm{~A}$ & $18 \mathrm{~b} \mathrm{~A}$ & 27 a $A$ & $9 \mathrm{~cd} \mathrm{~A}$ & $8 \mathrm{~cd} \mathrm{~A}$ \\
\hline & $91 / 92$ & $7 \mathrm{~cd} \mathrm{~A}$ & $12 \mathrm{bc} \mathrm{A}$ & $13 \mathrm{~b} \mathrm{~A}$ & 20 a $\mathrm{A}$ & 20 a B & $9 \mathrm{bcd} A$ & $6 \mathrm{~d} \mathrm{~A}$ \\
\hline & $92 / 93$ & $8 \mathrm{c} \mathrm{A}$ & $12 \mathrm{c} \mathrm{A}$ & $12 \mathrm{bc} \mathrm{A}$ & 19 a A & $17 \mathrm{ab} \mathrm{B}$ & $8 \mathrm{c} \mathrm{A}$ & $8 \mathrm{c} \mathrm{A}$ \\
\hline & Médias & 7 & 12 & 12 & 18 & 21 & 10 & 7 \\
\hline & Teste F: & \multirow{2}{*}{\multicolumn{2}{|c|}{$\begin{array}{l}\text { Tratamento }(\mathrm{T})=53,07 * * \\
\text { Tratamento }(\mathrm{T})=23,45\end{array}$}} & \multirow{2}{*}{\multicolumn{3}{|c|}{$\begin{array}{l}\text { A no (A) }=0,83^{\text {ns }} \\
\text { A no (A) }=18,02\end{array}$}} & $\bar{a} o(T \times A)=$ & $2,96 * *$ \\
\hline & C.V. $(\%)$ & & & & & & & \\
\hline \multirow{6}{*}{$\begin{array}{c}\mathrm{Fe} \\
\mathrm{mg} / \mathrm{kg}\end{array}$} & $89 / 90$ & 125 a $\mathrm{A}$ & $115 \mathrm{a} \mathrm{A}$ & 148 a $\mathrm{A}$ & 238 a B & $1.089 \mathrm{a} \mathrm{B}$ & 261 a B & $113 \mathrm{a} \mathrm{A}$ \\
\hline & $90 / 91$ & $132 \mathrm{c} \mathrm{A}$ & $140 \mathrm{c} \mathrm{A}$ & $145 \mathrm{c} \mathrm{A}$ & 3.456 a A & $2.573 \mathrm{ab} \mathrm{A}$ & 388 c B & $1.283 \mathrm{bc} \mathrm{A}$ \\
\hline & $91 / 92$ & $207 \mathrm{~b} \mathrm{~A}$ & $283 \mathrm{~b} \mathrm{~A}$ & $283 \mathrm{~b} \mathrm{~A}$ & $2.455 \mathrm{a} \mathrm{A}$ & $1.606 \mathrm{abAB}$ & 2.189 a A & $280 \mathrm{~b} \mathrm{~A}$ \\
\hline & $92 / 93$ & $805 \mathrm{~b} \mathrm{~A}$ & $373 \mathrm{~b} \mathrm{~A}$ & $1.250 \mathrm{~b} \mathrm{~A}$ & 2.948 a A & $1.328 \mathrm{~b} \mathrm{AB}$ & $2.851 \mathrm{a} \mathrm{A}$ & $973 \mathrm{~b} \mathrm{~A}$ \\
\hline & Médias & 317 & 228 & 456 & 2274 & 1648 & 1422 & 662 \\
\hline & $\begin{array}{l}\text { Teste F: } \\
\text { C.V.(\%) }\end{array}$ & \multicolumn{2}{|c|}{$\begin{array}{l}\text { Tratamento }(\mathrm{T})=21,91 * * \\
\text { Tratamento }(\mathrm{T})=66,98\end{array}$} & \multicolumn{3}{|c|}{$\begin{array}{l}\text { A no }(\mathrm{A})=14,19 * * \\
\text { A no }(\mathrm{A})=71,23\end{array}$} & $\mathrm{o}(\mathrm{T} \times \mathrm{A})=$ & $7 * *$ \\
\hline \multirow{6}{*}{$\begin{array}{c}\mathrm{Mn} \\
\mathrm{mg} / \mathrm{kg}\end{array}$} & $89 / 90$ & $47 \mathrm{~b} \mathrm{~A}$ & $30 \mathrm{~b} \mathrm{~B}$ & $54 \mathrm{ab} \mathrm{A}$ & $76 \mathrm{ab} \mathrm{B}$ & $150 \mathrm{a} \mathrm{B}$ & $87 \mathrm{ab} \mathrm{B}$ & $106 \mathrm{ab} \mathrm{A}$ \\
\hline & $90 / 91$ & $44 \mathrm{c} \mathrm{A}$ & 70 с A B & $43 \mathrm{c} \mathrm{A}$ & $234 \mathrm{ab} \mathrm{A}$ & $217 \mathrm{ab}$ AB & 264 a A & $137 \mathrm{bc} \mathrm{A}$ \\
\hline & $91 / 92$ & $70 \mathrm{~d} \mathrm{~A}$ & $119 \mathrm{~cd} \mathrm{~A}$ & $103 \mathrm{~cd} \mathrm{~A}$ & $220 \mathrm{ab} A$ & 287 a A & $241 \mathrm{ab} \mathrm{A}$ & $175 \mathrm{bc} \mathrm{A}$ \\
\hline & $92 / 93$ & $60 \mathrm{~b} \mathrm{~A}$ & $57 \mathrm{~b} \mathrm{AB}$ & $96 \mathrm{ab} \mathrm{A}$ & 159 a A B & 162 a B & $128 \mathrm{ab} \mathrm{B}$ & $98 \mathrm{ab} \mathrm{A}$ \\
\hline & Médias & 55 & 69 & 74 & 172 & 204 & 180 & 129 \\
\hline & $\begin{array}{l}\text { Teste F: } \\
\text { C.V. }(\%)\end{array}$ & \multicolumn{2}{|c|}{$\begin{array}{l}\text { Tratamento }(\mathrm{T})=25,42 * * \\
\text { Tratamento }(\mathrm{T})=38,20\end{array}$} & \multicolumn{3}{|c|}{$\begin{array}{l}\text { Ano }(\text { A })=23,46 * * \\
\text { Ano }(A)=35,81\end{array}$} & $\mathrm{o}(\mathrm{T} \times \mathrm{A})=$ & $66 * *$ \\
\hline \multirow{6}{*}{$\begin{array}{c}\mathrm{Zn} \\
\mathrm{mg} / \mathrm{kg}\end{array}$} & $89 / 90$ & $24 \mathrm{c} \mathrm{A}$ & $30 \mathrm{abcA}$ & $25 \mathrm{bc} \mathrm{A}$ & 32 a $\mathrm{A}$ & $31 \mathrm{ab} A B$ & 34 a $\mathrm{A}$ & $24 \mathrm{c} \mathrm{A}$ \\
\hline & $90 / 91$ & $16 \mathrm{~d} \mathrm{~B}$ & $22 \mathrm{~cd} \mathrm{~B}$ & $17 \mathrm{~d} \mathrm{~B}$ & $29 \mathrm{ab} \mathrm{A}$ & 36 a A & $28 \mathrm{bc} \mathrm{A}$ & $19 \mathrm{~d} \mathrm{~A}$ \\
\hline & $91 / 92$ & $21 \mathrm{c} \mathrm{AB}$ & $28 \mathrm{ab} \mathrm{A}$ & $22 \mathrm{bc} \mathrm{AB}$ & $27 \mathrm{abcA}$ & $27 \mathrm{abc} B$ & 32 a $A$ & $24 \mathrm{bc} \mathrm{A}$ \\
\hline & $92 / 93$ & $23 \mathrm{~b} \mathrm{~B}$ & $25 \mathrm{ab} \mathrm{AB}$ & $22 \mathrm{~b} \mathrm{~A} \mathrm{~B}$ & $28 \mathrm{ab} \mathrm{A}$ & $27 \mathrm{ab} \mathrm{B}$ & 30 a A & $22 \mathrm{~b} \mathrm{~A}$ \\
\hline & Médias & 21 & 26 & 21 & 29 & 30 & 31 & 22 \\
\hline & $\begin{array}{l}\text { Teste F: } \\
\text { C.V.(\%) }\end{array}$ & \multicolumn{2}{|c|}{$\begin{array}{l}\text { Tratamento }(\mathrm{T})=28,61 * * \\
\text { Tratamento }(\mathrm{T})=12,52\end{array}$} & \multicolumn{4}{|c|}{$\begin{array}{l}\text { Ano }(\mathrm{A})=10,01 * * \\
\text { Ano }(\mathrm{A})=12,05\end{array}$} & $8 * *$ \\
\hline
\end{tabular}

- Em cada ano (A), médias seguidas pela mesma letra maiúscula na vertical e em cada tratamento (T), médias seguidas de mesma letra minúscula na horizontal, não diferem entre si, pelo teste de Tukey, a 5\% de probabilidade ( $\mathrm{P}>0,05)$.

TABELA 5 - Quantidades médias de nutrientes incorporados ao solo pelo cultivo de leguminosas intercalares aos citros, nos anos agrícolas 1989/90 a 92/93, EECB, Bebedouro-SP

\begin{tabular}{|c|c|c|c|c|c|c|c|c|c|c|c|}
\hline \multirow{2}{*}{ TRA TA MENTO } & \multicolumn{6}{|c|}{ MACRONUTRIENTES $(\mathrm{kg} / \mathrm{ha})$} & \multicolumn{5}{|c|}{ MICRONUTRIENTES $\quad(\mathrm{g} / \mathrm{ha})$} \\
\hline & $\mathrm{N}$ & $\mathrm{P}_{2} \mathrm{O}_{5}$ & $\mathrm{~K}_{2} \mathrm{O}^{* *}$ & $\mathrm{Ca}$ & $\mathrm{Mg}$ & $\mathrm{S}$ & $\mathrm{B}$ & $\mathrm{Cu}$ & $\mathrm{Fe}$ & $\mathrm{Mn}$ & $\mathrm{Zn}$ \\
\hline C.juncea & 183,4 & 39,2 & 204,4 & 104,8 & 52,4 & 13,1 & 236 & 92 & 4.153 & 721 & 275 \\
\hline C. spectabilis & 44,3 & 10,2 & 56,1 & 38,4 & 9,8 & 3,4 & 74 & 30 & 561 & 170 & 64 \\
\hline Mucuna-preta & 85,6 & 18,8 & 72,6 & 39,2 & 14,2 & 6,4 & 93 & 64 & 8.095 & 612 & 103 \\
\hline Mucuna-anã & 91,0 & 15,3 & 54,6 & 31,5 & 14,0 & 7,0 & 91 & 74 & 5.768 & 714 & 105 \\
\hline Labe-labe & 67,4 & 19,2 & 69,3 & 41,7 & 19,3 & 7,1 & 93 & 32 & 4.565 & 578 & 100 \\
\hline
\end{tabular}

* - Quantidade de nutrientes considerando plantio das leguminosas em área total. Neste experimento, considerar $50 \%$ dos valores acima, ou seja, área intercalar dos citros;

** - Valores de $\mathrm{P}$ e $\mathrm{K}$ transformados ao equivalente em kg/ha de $\mathrm{P}_{2} \mathrm{O}_{5}$ e $\mathrm{K}_{2} \mathrm{O}$. Fator de conversão para $\mathrm{P}=2,3$ e $\mathrm{K}=1,2$. 
apresentava mais pobre em nutrientes e que a reciclagem, a partir deste ano, melhorou a fertilidade e as condições para o desenvolvimento das leguminosas plantadas posteriormente.

Observa-se, na Tabela 2, que a Crotalaria juncea se destacou na produção média de material seco $(13,1)$, seguida pelo guandu $(6,84)$, feijão-de-porco $(6,05)$, mucuna-preta $(3,56)$, mucuna-anã $(3,50)$, labe-labe $(3,21)$ e Crotalaria spectabilis $(2,46$ t/ha). Os valores médios de material produzidos em t/ha, estão próximos aos obtidos por Kiehl (1980), Azeredo \& Manhães (1983) e IAPAR (1984). Devido à lenta germinação, a C. spectabilis, sofreu competição com o mato, tendo sua produção comprometida; porém, nos anos seguintes, houve uma menor incidência de mato e aumento da densidade populacional, melhorando a produção de material verde e cobertura do solo. Já a mucuna-anã sofreu ataque do fungo da cercosporiose, resultando em queda precoce de folhas e menores valores de material verde, principalmente no ano de 1990/91, produzindo 3,93 t/ha aos 148 dias após o plantio, enquanto, nos anos com incorporação entre 113 e 106 dias, a produção variou entre 15,7 e $31,8 \mathrm{t} / \mathrm{ha}$. Os valores apresentados na Tabela 2 mostram a produção considerando o plantio em área total; porém, no caso do cultivo intercalar ao citros, devem-se considerar apenas 50\% dos valores de produção de fitomassa, pois o plantio das leguminosas restringiu-se a $50 \%$ da área.

O guandu e a mucuna-preta, espécies de ciclo longo com florescimento após os 140 dias quando avaliadas aos 148 dias da semeadura, apresentaram maiores valores de material verde, quando comparados com as demais espécies de ciclo curto, em torno dos 100 a 110 dias. Já a C. juncea, apesar de florescer aos 110 dias, aos 148, apresentava-se mais fibrosa e, por isso, com o maior peso da matéria verde $(59,1 \mathrm{t} / \mathrm{ha})$. Isto se confirma na avaliação dos nutrientes presentes na matéria verde nas mesmas datas da avaliação, mostrando menores teores de $\mathrm{N}, \mathrm{P}, \mathrm{Ca}, \mathrm{Mg}$, $\mathrm{B}, \mathrm{Cu}, \mathrm{Mn}$ e $\mathrm{Zn}$, conforme ilustram as Tabelas 3 e 4. Polli \& Chada (1989) observaram que há uma certa relação entre plantas mais tenras e o maior teor de $\mathrm{N}$ na parte aérea.

De acordo com dados apresentados por Azeredo \& Manhães (1983), a C. spectabilis e a mucuna-preta produziram valores inferiores de matéria fresca da parte aérea, estando as demais espécies estudadas dentro da média citada.

Em geral, quando as plantas foram avaliadas aos 148 dias (1990/91), todas as espécies apresentaram menores teores de nutrientes na parte aérea. Isto leva a crer que ocorre um carreamento de nutrientes para as sementes e transformações bioquímicas, tornando o material mais fibroso, de difícil degradação microbiológica e mais pobres em nutrientes (Mello, 1978).

Os adubos verdes devem ser incorporados ao solo, de preferência, após o florescimento e antes da frutificação, para garantir a adição de uma grande quantidade de material vegetal. A incorporação das plantas, após o desenvolvimento dos frutos, vai resultar no uso de um material mais pobre e possível infestação dos solos com as sementes do adubo verde (Costa, 1989).

A análise química da parte aérea das leguminosas, conforme ilustra a Tabela 3, revelou que, de modo geral, as espécies lenhosas (C. juncea, C. spectabilis, guandu e feijãode-porco) apresentaram menores teores de nutrientes; já as herbáceas e rasteiras (mucuna-preta, mucuna-anã e labe-labe) foram as espécies que apresentaram os maiores teores, principalmente $\mathrm{N}, \mathrm{S}$ e $\mathrm{P}$, elementos estes fundamentais na formação de proteínas. Quando as leguminosas foram incorporadas aos 148 dias (ano agrícola 1990/91), ao contrário dos demais anos (aproximadamente 110 dias), observou-se diminuição dos teores médios de N, P, Ca, Mg, B e Zn. Esta ocorrência também foi relatada por Castro (1956), que comprovou, nos primeiros meses do período vegetativo, ocorrência de mais alta porcentagem de $\mathrm{N}$ nos tecidos das leguminosas, mas a maior quantidade de nutrientes é encontrada na época do florescimento, sendo o momento mais oportuno para o corte, pois as folhas e os talos tenros que constituem as partes mais fáceis para decomposição, são atacados pelos microrganismos e começam a formar amônio e nitratos utilizáveis pelas plantas.

A $C$. juncea apresentou os menores teores de nutrientes no material vegetal; já o labe-labe apresentou, em média, os maiores teores de P, Mg, S, B e Zn, e o feijão-de-porco destacouse por apresentar os maiores teores de $\mathrm{N}, \mathrm{K}$ e Ca na parte aérea quando comparado com as demais espécies.

De um modo geral, o feijão-de-porco e o labe-labe foram as espécies que apresentaram melhores teores médios de macronutrientes na parte aérea, seguidos pela mucuna-preta que se destacou nos teores de $\mathrm{N}$ e $\mathrm{P}$, a $C$. spectabilis para $\mathrm{K}$ e Ca e a mucuna-anã para $\mathrm{N}$ e $\mathrm{S}$.

Ambrosano et al. (1997) determinaram teores de $\mathrm{N}$ de 27,6 e $16,8 \mathrm{~g} / \mathrm{kg}$ na parte aérea da mucuna-preta e da $C$. juncea, respectivamente, valores bastante próximos aos obtidos neste experimento, com média de quatro anos de 24 e $14 \mathrm{~g} / \mathrm{kg}$, respectivamente.

A incorporação aos 148 dias reduziu também os teores médios de B e Zn na parte aérea das leguminosas (Tabela 4), indicando que a incorporação tardia incorpora um material mais pobre também em micronutrientes. Os maiores teores de micronutrientes foram encontrados na parte aérea da mucunaanã (Cu, Mn e Zn), já a C. spectabilis maiores de B e a mucunapreta maiores de Fe.

De posse dos resultados de produção média de material verde (Tabela 2) e teores médios de nutrientes (Tabelas 3 e 4), pode-se calcular a quantidade de nutrientes incorporados ao solo pelo cultivo das leguminosas (Tabela 5). Assim, verifica-se que as espécies estudadas são grandes incorporadoras e recicladoras de nutrientes ao solo, considerando apenas a parte aérea, onde se destacam a $C$. juncea, guandu e feijão-de-porco com valores significativos de N (183,4; 143,6 e 169,4 kg/há, respectivamente), concordando com valores obtidos por Mello (1978). Segundo Igue (1984), 2/3 do N total das leguminosas é proveniente da fixação simbiótica de $\mathrm{N}_{2}$ atmosférico, e o restante é absorvido do solo numa quantidade total de $\mathrm{N}$ que varia de 15 a $30 \mathrm{~g} / \mathrm{kg}$ do resíduo orgânico.

Estudando a dinâmica do nitrogênio na crotalária e na mucuna-preta marcadas com 15N, Ambrosano (1995) determinou que 60 a $80 \%$ do $\mathrm{N}$ das leguminosas permaneceu no solo, 20 a 30 $\%$ foi absorvido pelas plantas de milho e que 5 a $15 \%$ deixou o sistema solo-planta.

Os $183,4 \mathrm{~kg} / \mathrm{ha}$ de $\mathrm{N}$ incorporados pela $C$. juncea, considerando plantio em área total, correspondem a $91,7 \mathrm{~kg} / \mathrm{ha}$ para plantio na área intercalar dos citros (50\%), ou seja, equivale à aplicação de $204 \mathrm{~kg}$ de uréia/ha ou $460 \mathrm{~kg}$ de sulfato de amônia/ ha na área intercalar. 
Os demais macros e micronutrientes são provenientes da reciclagem de nutrientes efetuada por estas espécies, que absorvem os nutrientes lixiviados no perfil do solo (camadas subsuperficiais principalmente), trazendo-os novamente à superfície do solo onde estarão disponíveis às culturas econômicas. Este processo de incorporação de nutrientes lixiviados, nas camadas superficiais, se aplica muito bem à cultura de citros, que apresenta, segundo Rodrigues (1980), concentração das raízes nos 40 e $60 \mathrm{~cm}$ de profundidade.

Carvalho et al. (1999) demonstraram que a distribuição do sistema radicular da laranjeira-'Pêra' no perfil do solo foi influenciada positivamente pela melhoria da estrutura do solo, ocasionada pelo manejo do feijão-de-porco associado à subsolagem na profundidade média de $55 \mathrm{~cm}$, o que resultou em melhor distribuição do sistema radicular dos citros em busca de água e nutrientes.

A C. spectabilis e a mucuna-anã incorporaram menores quantidade de nutrientes ao solo, provavelmente devido à baixa germinação da primeira e suscetibilidade à cercosporiose da segunda. Como incorporador de cálcio, o feijão-de-porco destacou-se com a média de 108,9 kg/ha.

\section{CONCLUSÕES}

1. As espécies leguminosas estudadas desenvolvem satisfatoriamente intercalar ao citros sem necessidade de manejo pós-plantio, promovendo reciclagem e incorporação de quantidades significativas de nutrientes ao solo, destacandose o N, K, Ca e P, possibilitando substituir ou reduzir uma parcela dos adubos nitrogenados químicos nos citros.

2. É recomendado alternar espécies leguminosas nas ruas dos citros de acordo com o porte, facilitando manejar os citros no período.

3. Maiores valores de biomassa e reciclagem de nutrientes foram obtidos com a Crotalaria juncea, guandu e feijão-de-porco.

4. O guandu e o labe-labe permitem corte alto, pois rebrotam satisfatoriamente, podendo ser manejados por mais de um ano agrícola.

\section{REFERÊNCIAS BIBLIOGRÁFICAS}

AMBROSANO, E.J. Dinâmica do nitrogênio dos adubos verdes, crotalária juncea (Crotalaria juncea) e mucuna-preta (Mucuna aterrima), em dois solos cultivados com milho. Piracicaba, 1995. 83p. Tese (Doutorado) - Escola Superior de Agricultura "Luiz de Queiroz", Universidade São Paulo, Piracicaba, 1995.

AMBROSANO, E.J.; TRIVELIN, P.C.O.; MURAOKA, T. Técnica para marcação dos adubos verdes crotalária juncea e mucunapreta com ${ }^{15} \mathrm{~N}$ para estudos de dinâmica do nitrogênio. Bragantia, Campinas, v. 56, n.1, p. 219-24, 1997

AZEREDO, F.D.; MANHÃES, M.S. Adubação orgânica. In: ORLANDOFILHO, J.(Ed). Nutrição e adubação da cana-de-açúcar no Brasil. Piracicaba: Planalsucar, 1983. p.211-25.
CARVALHO, J.E.B.; SOUZA, L.S.; JORGE, L.A.C.; RAMOS, W.F., NETO, A.O.C., ARAÚJO, A.M.A., LOPES, L.C.; JESUS, M.S. Manejo de coberturas do solo e sua interferência no desenvolvimento do sistema radicular da laranja-'Pêra'. Revista Brasileira de Fruticultura, Jaboticabal, v.21, n.2, p. 140-5, 1999.

CASTRO, F.S. Conservación de suelos. Barcelona: Salvat, 1956. 298p.

COSTA, M.B.B. Adubação orgânica: nova síntese e novo caminho para a agricultura. São Paulo: Icone, 1989. 107p. (Coleção Brasil Agrícola)

IAPAR. Adubação orgânica. Relatório técnico anual, 1982. Londrina, 1984, 326p.

IGUE, K. Dinâmica da matéria orgânica e seus efeitos nas propriedades do solo. In: Adubação verde no Brasil. Campinas: Fundação Cargill, 1984, p. 232-67.

KIEHL, E.J. Leucena: alternativa para pequena e média agricultura. Goiânia: EMBRAPA-CNPAF, 1980.23p. (Circular Técnica, 6)

MALAVOLTA, E.; VITTI, G.C.; OLIVEIRA, S.A. de. Avaliação do estado nutricional das plantas: princípios e aplicações. Piracicaba SP: Associação Brasileira para Pesquisa da Potassa e do Fosfato, 1989.201p.

MELLO, F.A.F. Fixação de nitrogênio por algumas leguminosas. Revista de Agricultura, Piracicaba, v.53, 1978. p, 59-63.

MIYASAKA, S.; GALLO, J.R.; SILVA, J.G. Histórico de estudos de adubação verde, leguminosas viáveis e suas características. In: Adubação verde no Brasil, Campinas: Fundação Cargill, 1984. p.64-123.

POLLI, H.; CHADA, S. Adubação verde ou em cobertura na produção de milho em solo de baixo potencial de produtividade. Revista Brasileira de Ciência do Solo, Campinas, v.13, n. 3, p. 287-93, 1989.

RODRIGUEZ, O. Nutrição e adubação dos citros. In: RODRIGUEZ, O. \& VIÉGAS, F. Citricultura brasileira, Campinas: Fundação Cargill, 1980.v. 2, p. 387-430.

RUSSELL, R.S.;IGUE, K.; MEHTA, Y.R. The soil-root system in relation to brazilian agriculture. Londrina, IAPAR, 1981.372p.

SILVA, J.A. A consorciação de adubos verdes na cultura de citros em formação. Piracicaba, 1995. 116p. Dissertação (Mestrado) - Escola Superior de Agricultura "Luiz de Queiroz", Universidade São Paulo, Piracicaba, 1995.

SILVA, J.A.A.; DONADIO, L.C.; CARLOS, J.A.D. Adubação verde em citros. Jaboticabal: FUNEP, 1999. 37p. (Boletim Citrícola, 9). 